\title{
Complex-Mass Definition and the Structure of Unstable Particle's Propagator
}

\author{
Vladimir Kuksa \\ Research Institute of Physics, Southern Federal University, Pr. Stachky 194, Rostov-on-Don 344090, Russia \\ Correspondence should be addressed to Vladimir Kuksa; vkuksa47@mail.ru
}

Received 30 July 2015; Revised 28 October 2015; Accepted 3 November 2015

Academic Editor: Shi-Hai Dong

Copyright ( 2015 Vladimir Kuksa. This is an open access article distributed under the Creative Commons Attribution License, which permits unrestricted use, distribution, and reproduction in any medium, provided the original work is properly cited. The publication of this article was funded by SCOAP S $^{3}$

\begin{abstract}
The propagators of unstable particles are considered in framework of the convolution representation. Spectral function is found for a special case when the propagator of scalar unstable particle has Breit-Wigner form. The expressions for the dressed propagators of unstable vector and spinor fields are derived in an analytical way for this case. We obtain the propagators in modified Breit-Wigner forms which correspond to the complex-mass definition.
\end{abstract}

\section{Introduction}

Two standard definitions of the mass and width of unstable particles (UP), which are usually considered in the literature, have different nature. The on-mass-shell (OMS) scheme defines the mass $M$ and width $\Gamma$ of UP by the renormalization of the self-energy amplitude. In the pole scheme (PS), the definitions of mass and width are based on the complexvalued position of the propagator pole $s_{R}-M_{0}^{2}-\Pi\left(s_{R}\right)=0$. There has been considerable discussion concerning definition of the vector-boson mass [1-11]. It was shown that OMS scheme contains spurious higher-order gauge-dependent terms. Moreover, at one loop in the conventional OMS, the problem of threshold singularity arises which originates from the wave-function renormalization constant $Z^{-1}=1-$ $\Re A^{\prime}\left(M^{2}\right)$ [6]. The PS provides gauge invariant definition and make it possible to solve the problem of threshold singularity $[6,7]$. However, it does not define the mass and width in unique way [12]. One of the PS definitions, where mass $M_{\rho}$ and width $\Gamma_{\rho}$ follow from the parametrization $s_{R}=M_{\rho}^{2}-$ $i M_{\rho} \Gamma_{\rho}[12]$, is known as complex-mass definition. It should be noted that the PS definition of the mass and width is connected with the structure of the dressed propagator. Some aspects of the abovementioned problems are considered further in more detail.
Traditional way to construct the dressed propagator of UP is the Dyson summation which introduces the width and redefines the mass of UP. This procedure runs into some problems which are widely discussed in the literature. One of such problems follows from the d'Alembert convergence criterion $|z|<1$ of the series

$$
\frac{1}{1-z}=\sum_{k=0}^{\infty} z^{k}=1+z+z^{2}+\cdots, \quad|z|<1,
$$

where $z=\Pi_{(1)}(q) /\left(q^{2}-M_{0}^{2}\right)$ and $\Pi_{(1)}(q)$ is the oneparticle-irreducible self-energy. The variable $z$ should be correctly redefined before summation; that is, we have to perform the renormalization of the $\Pi_{(1)}(q)$ at Lagrangian level. This procedure must be consistent with the infinite Dyson summation and we cannot use it after the redefinition at $|z|>1$. For instance, in the resonant-part approximation we can define $z=\Im \Pi_{(1)}(q) /\left(q^{2}-M^{2}\right) \approx M \Gamma /\left(q^{2}-\right.$ $\left.M^{2}\right)$. So, the peak range $\left|q^{2}-M^{2}\right|<M \Gamma$ or $|q-M|<$ $\Gamma / 2($ at $\Gamma \ll M)$ is excluded by d'Alembert convergence criterion. There are, also, the difficulties in the scheme of sequential fixed-order calculations which exhibit themselves in the violation of the gauge invariance. Moreover, using different decompositions of self-energy tensor in the Dyson summation leads to different expressions for vector dressed 
propagator [13-15]. Then, the renormalization procedure is connected with the truncation of a Laurent series expansion at the resonance range. So, the renormalized propagator is an approximation of the full one which corresponds to exact two-point function.

The peculiarities of Dyson summation lead to the lack of uniqueness in constructing the propagators of unstable particles. There are several different expressions for the numerator of vector-boson propagator $g_{\mu \nu}-q_{\mu} q_{\nu} / f(q, M, \Gamma)$, which are exploited in practical calculations and give almost the same numerical results. The denominator $f(q, M, \Gamma)$ has the following forms (in the unitary gauge): $M^{2}, M^{2}-i M \Gamma$, $(M-i \Gamma / 2)^{2}, q^{2}$, and other combinations with $q$-dependent $M, \Gamma$. So, we need in an additional argumentation concern these semiphenomenological definitions. It is known that the commonly used Breit-Wigner (BW) expressions for bosonic and fermionic propagators, respectively,

$$
\begin{aligned}
D_{\mu \nu}^{V}\left(q^{2}\right) & =\frac{-g_{\mu \nu}+q_{\mu} q_{\nu} / M_{V}^{2}}{q^{2}-M_{V}^{2}+i M_{V} \Gamma_{V}}, \\
D_{F}(\hat{q}) & =\frac{\widehat{q}+M_{F}}{q^{2}-M_{F}^{2}+i M_{F} \Gamma_{F}}
\end{aligned}
$$

do not satisfy the electromagnetic Ward identity [3]. It was shown in $[3,13,14]$ that the modified BW propagators,

$$
\begin{aligned}
D_{\mu \nu}^{V}\left(q^{2}\right) & =\frac{-g_{\mu \nu}+q_{\mu} q_{\nu} /\left(M_{V}^{2}-i M_{V} \Gamma_{V}\right)}{q^{2}-\left(M_{V}^{2}-i M_{V} \Gamma_{V}\right)}, \\
D_{F}(\hat{q}) & =\frac{\widehat{q}+M_{F}-i \Gamma_{F} / 2}{q^{2}-\left(M_{F}-i \Gamma_{F} / 2\right)^{2}},
\end{aligned}
$$

satisfy the electromagnetic Ward identity which provides the gauge invariant description of processes with UP participation. Note, here we deal with the resonant part of the full propagator which follows from Dyson summation. The correctness of this propagator should be understood in the context of resonant processes where the usage of the modified BW propagator leads consistently to gauge invariant results $[3,13,14]$. It was also noted in [13] that, in this case, we have to make the modification $M_{V}^{2} \rightarrow M_{V}^{2}-i M_{V} \Gamma_{V}$ not only in the $q_{\mu} q_{\nu}$ term of the propagator, but in the vertexes also. Thus, we get the so-called complex-mass definition which was developed in the framework of the complex-mass scheme (CMS) [16-20]. Recently, BW parametrization of the resonance lines has been developed in the works [21-23]. In particular, a new parameter has been introduced into this parametrization which describes a fundamental property of a resonance [23].

All abovementioned definitions are connected with the structure of dressed propagators which follows from Dyson summation. As was noted above, this procedure runs into some problems which are widely discussed in literature. An alternative approach is based on the spectral representation of the propagator of UP. It has a long history [24-34] and treats UP as a nonperturbative state or effective field (asymptotic free field [30, 31]). For the first time, the hypothesis of continuous (smeared) mass of UP was suggested by Matthews and Salam [27]. In this paper, they have formulated “...a very direct interpretation to the spectral function introduced by Kallen [26] and Lehmann [24]." The authors interpreted the spectral function as "...distribution of mass values, with a spread, $\delta m$, related to the mean life $\delta \tau(=1 / \lambda)$, by uncertainty relation $\delta m \delta \tau \sim 1$ " (see Introduction in [27]). In $[30,31]$, UP is described by the so-called asymptotic free field as the state with indefinite (not fixed) mass. The hypothesis of continuous (smeared, indefinite) mass of UP was developed in a series of works, where a quantum field model of UP was presented (see, e.g., review articles $[35,36]$ and references therein). In this approach, the physical values of the mass and width are related to the parameters of continuous mass distribution. It should be noted that the definition of the spectral function does not follow from the first principles. So, it was constructed in phenomenological ways and has a different form in abovementioned papers. Moreover, the spectral function is sensible to the "tails" of distribution.

In this work, we consider the structure of the propagators in the framework of the spectral-representation approach and on account of the Dyson procedure. As was noted early, Dyson summation is not well-defined at peak range, while the spectral approach cannot be applied far from the peak. So, we have used the information which follows from both approaches in the domains of their validity. We suppose that the propagator of scalar UP in spectral representation coincides with the BW one in the intersection of their domains of definition. Using this assumption we define the spectral function of boson UP and apply it for the case of the vector UP's propagator. We show that this strategy strictly leads to the propagators which have the structure of the modified Breit-Wigner ones (3) under the condition that the spectral function is defined for both positive and negative values of its parameter (see Section 2).

The paper is organized as follows. In Section 2 we present the principal elements of the approach and analyze the general structure of the scalar propagator. The expressions for vector and fermionic propagators are derived in Sections 3 and 4, respectively. Some aspects of Lagrangian formalism with unstable field ingredient and respective conclusions concerning the physical status of the results are made in Sections 5 and 6.

\section{Propagator of Scalar Unstable Particle}

The structure of propagator for the case of scalar UP can be represented in the following convolution form:

$$
\begin{aligned}
D(q) & =i \int_{s_{0}}^{\infty} \frac{\rho\left(m^{2}\right) d m^{2}}{q^{2}-m^{2}+i \epsilon} \\
& =i \int_{s_{0}}^{\infty} D_{0}\left(q^{2}, m^{2}\right) \rho\left(m^{2}\right) d m^{2},
\end{aligned}
$$

where $\rho\left(m^{2}\right)$ is spectral function of the parameter $m^{2}$, $D_{0}\left(q^{2}, m^{2}\right)$ is "bare" scalar propagator, and the limit of integration $s_{0}$ will be determined further. Note that the symbolic expression (4) has different explicit form in various approaches. One can get a traditional Lehmann-Kallen 
representation for the case $\rho\left(m^{2}\right)=\delta\left(M^{2}-m^{2}\right)+\rho_{\mathrm{LK}}\left(m^{2}\right)$, where $\rho_{\mathrm{LK}}\left(m^{2}\right)=0$ below the threshold $m^{2}<4 M^{2}$. In the framework of the asymptotic free field approaches (indefinite mass) $[30,31]$ or the model with continuous mass $[35,36]$ the expression (4) can be derived directly. In these cases, the field function of scalar UP can be represented in the following convolution form:

$$
\phi(x)=\frac{1}{(2 \pi)^{3 / 2}} \iint \phi\left(\mathbf{p}, m^{2}\right) e^{i p x} d \mathbf{p} \omega\left(m^{2}\right) d m^{2},
$$

where $p=\left(\mathbf{p}, p^{0}\right), \phi\left(\mathbf{p}, m^{2}\right)$ is defined in standard way at fixed mass $p^{2}=m^{2}$ and $\omega\left(m^{2}\right)$ is model weight function. Note that the value $m$ is not a conventional observed mass of UP. It is continuous mass parameter which cuts out threedimensional surface in the four-momentum space according to equality $p^{2}=m^{2}$. The canonical commutation relations contain an additional delta-function $\delta\left(m^{2}-m^{\prime 2}\right)$. Starting from the standard definition of Green's function $D(q)=$ $i \int d x \exp (-i q x)\langle 0|\widehat{T} \phi(x) \phi(0)| 0\rangle$, where $\phi(x)$ is defined by (5), by straightforward calculations we get convolution representation of the model propagator $(4)$, where $\rho\left(m^{2}\right)=$ $\left|\omega\left(m^{2}\right)\right|^{2}$.

The principal problem of the approach under consideration is to define the spectral function $\rho\left(m^{2}\right)$. In this connection we should note the general peculiarity of the spectral approaches. From expression (4) the problem with threshold value of the spectral parameter $s_{0}$ follows. Propagator of UP near the threshold $q^{2} \approx s_{0}$ contains divergent at $q^{2} \rightarrow s_{0}$ contributions which are compensated far from the threshold. This threshold effect is explicitly described with the help of the known integration rule

$$
\int_{a}^{b} \frac{f(x) d x}{x \pm i \epsilon}=\mp i \pi f(0)+\mathscr{P} \int_{a}^{b} \frac{f(x)}{x} d x,
$$

which follows from the Sokhotski-Plemelj formula when $x=$ $0 \in(a, b)$. In $(6) \mathscr{P} \int$ stands for the Cauchy principal value of the integral. The threshold effect is caused by the pole at $q^{2}=s_{0}$ and will be described further in more detail.

Here, we consider the special case of the spectral function for scalar UP in the assumption that the scalar propagator has a conventional $\mathrm{BW}$ form:

$$
D^{\mathrm{BW}}(q)=\frac{1}{q^{2}-M^{2}+i M \Gamma} .
$$

In (7), we use $q$-independent scheme of the width insertion and omit general factor $i$ for simplicity. Scalar propagator in this form can be derived by Dyson summation too, if we use renormalization conditions $\mathrm{M}_{0}^{2}=\mathrm{M}^{2}-\mathfrak{R} \Pi\left(\mathrm{M}^{2}\right)$, $Z^{-1}=1+\mathfrak{R} \Pi^{\prime}\left(\mathrm{M}^{2}\right)$, and unitary condition $Z \mathfrak{I} \Pi\left(M^{2}\right)=$ $-\sqrt{M^{2}} \Gamma\left(M^{2}\right)[2,14]$ (note that the definitions of the $\Pi\left(\mathrm{M}^{2}\right)$ in these references have different sign). Taking into account the abovementioned peculiarities of summation procedure, we use expression (7) as phenomenological postulate which was verified by the experiments with good accuracy. Note also that expression (7) has the same status in the framework of CMS which does not rely on the Dyson procedure. Starting from the BW expression (7) for scalar propagator, we will define the corresponding spectral function $\rho\left(\mathrm{m}^{2}\right)$ and apply it to derive the expressions for the propagators of vector and spinor UP.

To define $\rho\left(m^{2}\right)$ we rewrite (4) with the help of the integration rule (6). Then, (4) takes the form:

$$
D(q)=-i \pi \rho\left(q^{2}\right)+\mathscr{P} \int \frac{\rho\left(m^{2}\right)}{q^{2}-m^{2}} d m^{2} .
$$

The condition $D(q)=D^{\mathrm{BW}}(q)$ leads to a following equalities:

$$
\begin{aligned}
& \Im D(q)=-\pi \rho\left(q^{2}\right)=\frac{-M \Gamma}{\left(q^{2}-M^{2}\right)^{2}+M^{2} \Gamma^{2}}, \\
& \Re D(q)=\mathscr{P} \int \frac{\rho\left(m^{2}\right) d m^{2}}{q^{2}-m^{2}}=\frac{q^{2}-M^{2}}{\left(q^{2}-M^{2}\right)^{2}+M^{2} \Gamma^{2}},
\end{aligned}
$$

where the first equalities follow from (8) and the second ones from (7). From the upper equality in (9) it follows that

$$
\rho\left(m^{2}\right)=\frac{1}{\pi} \frac{M \Gamma}{\left(m^{2}-M^{2}\right)^{2}+M^{2} \Gamma^{2}} .
$$

Thus, the condition $\Im D(q)=\mathfrak{\Im} D^{\mathrm{BW}}(q)$ uniquely defines the form of the function $\rho\left(\mathrm{m}^{2}\right)$ for the case under consideration ( $q$-independent $M$ and $\Gamma$ ). In [29] the definition of the function $\rho\left(\mathrm{m}^{2}\right)$ was given in close analogy with above consideration and was finished at this stage. Here, we take into consideration the lower equality of (9) which gives an additional information about the limits of integration. By straightforward calculation we can check that the lower equality of (9) and normalization of the function (10) are fulfilled exactly if $\left(-\infty<m^{2}<\infty\right)$. Inserting the expression (10) into the lower equality of (9) we get

$$
\begin{aligned}
& \Re \mathcal{R}(q)=\frac{M \Gamma}{\pi} \\
& \cdot \mathscr{P} \int_{s_{0}}^{\infty} \frac{d m^{2}}{\left(q^{2}-m^{2}\right)\left[\left(m^{2}-M^{2}\right)^{2}+M^{2} \Gamma^{2}\right]} \\
& \quad=\frac{M \Gamma}{\pi Q}\left[\frac{\pi p}{2 d}+\frac{1}{2} \ln \frac{\left(q^{2}-s_{0}\right)^{2}}{\left(q^{2}-s_{0}\right)^{2}-p\left(q^{2}-s_{0}\right)+Q}\right. \\
& \left.+\frac{p}{d} \arctan \frac{M^{2}-s_{0}}{M \Gamma}\right],
\end{aligned}
$$

where $Q=\left(q^{2}-M^{2}\right)^{2}+M^{2} \Gamma^{2}, p=2\left(q^{2}-M^{2}\right)$, and $d=2 M \Gamma$. From expression (11) it follows that the lower equality (9) is exact when $s_{0}=-\infty$. So, the parameter $m^{2}$ can take a negative value and we have to consider an analytic continuation of the traditional spectral approach. On the other hand, the expression (11) explicitly describes abovementioned threshold effect. The second term gives a logarithmic singularity at 
$q^{2} \rightarrow s_{0}$, where the point $q^{2}=s_{0}$ is cut out by the integration rule (6). The nature of this singularity differs from the one of the conventional threshold singularity, which takes place in OMS renormalization scheme [6, 7]. In the framework of the traditional Lehmann-like spectral approach, threshold effect is absent from the very beginning, because of $\rho\left(m^{2} \leq s_{0}\right)=0$. At the same time, BW form of scalar propagator cannot be reproduced exactly in this case. In our approach, $\rho\left(m^{2}\right)>0$ on the whole real axis, the threshold effect disappears at $s_{0} \rightarrow$ $-\infty$, and BW form is reproduced exactly. It should be noted that this effect has rather unphysical artificial nature (see, also, comments in Section 5).

Let us consider the theoretical status of the result and possible consequences of the presence of negative mass parameter $m^{2}<0$ in the integral representations (4) and (5). The condition $D(q)=D^{\mathrm{BW}}(q)$ defines the integral equation which contains the unknown function $\rho\left(\mathrm{m}^{2}\right)$ and was solved exactly. The form of the spectral function is defined strictly by the choice of the dressed scalar propagator as input condition. It should be noted that appearance of the negative component can be caused by the choice of the BW approximation. However, we do not know correct (exact) expression for the input propagator and evaluate the error of approximation. In the framework of the approaches with continuous mass, the negative component $m^{2}<0$ leads to the states with imaginary mass parameters which are usually interpreted as tachyons. The problem of existence of tachyons is under considerable discussion in the last decades. The main attention is paid to the principal problems such as violation of causality, tachyon vacuum, and radiation instability. It should be noted that these problems are related to UP as an observable object with fixed imaginary mass. In the framework of the effective model [36] UP is described by the positive mass square $M^{2}$ and we have no tachyons in the set of physical states.

Now we evaluate the contribution of the negative component. The spectral function $\rho\left(\mathrm{m}^{2}\right)$ is normalized and can be interpreted as the probability density of parameter $m^{2}$. So, the probability of the negative component is as follows:

$$
\begin{aligned}
P\left(m^{2}<0\right)=\int_{-\infty}^{0} \rho\left(m^{2} ; M, \Gamma\right) d m^{2} \approx & \frac{\Gamma}{\pi M}, \\
& \left(\frac{\Gamma}{M} \ll 1\right) .
\end{aligned}
$$

From (12) it follows that this probability is proportional to the factor $\Gamma / M$ which defines the finite-width effects in the processes with UP's participation. This fact can lead to an interesting possible conclusions: tachyon instability is intrinsic property of UP; it can be interpreted as the cause of unstable particle decay. Now, we evaluate the relative contribution of the negative component to the full propagator which we define as the following relation:

$$
\epsilon\left(q^{2}\right)=\frac{\int_{-\infty}^{0} D_{0}\left(q^{2}, m^{2}\right) \rho\left(m^{2}\right) d m^{2}}{\int_{-\infty}^{+\infty} D_{0}\left(q^{2}, m^{2}\right) \rho\left(m^{2}\right) d m^{2}} .
$$

In expression (13) denominator is full BW propagator (7) and the integration in numerator can be performed directly at $q^{2}>0$. As a result, we get

$$
\begin{aligned}
& \epsilon\left(q^{2} ; M, \Gamma\right)=\frac{1}{\pi} \\
& \cdot \frac{\Gamma M}{q^{2}-M^{2}-i \Gamma M}\left[\frac{1}{2} \ln \frac{q^{4}}{M^{2}\left(M^{2}+\Gamma^{2}\right)}\right. \\
& \left.+\pi \frac{q^{2}-M^{2}}{\Gamma M}\right]
\end{aligned}
$$

where we used the approximation $\arctan (M / \Gamma) \approx \pi / 2$ in the second term. From (14) strong $q^{2}$-dependence of the relative contribution $\epsilon\left(q^{2} ; M, \Gamma\right)$ follows. In particular, at the peak range $\epsilon\left(M^{2} ; \Gamma, M\right) \approx-i \Gamma^{2} / 2 \pi M^{2}$, at $q^{2} \gg M^{2}$ it has asymptotic $\epsilon\left(q^{2}\right) \rightarrow 1$, and at $q^{2} \ll M^{2}$ from (13) it follows that

$$
\epsilon\left(q^{2} ; M, \Gamma\right)=\frac{\Gamma}{\pi M}\left[\frac{1}{2} \ln \frac{M^{2}\left(M^{2}+\Gamma^{2}\right)}{q^{4}}+\pi \frac{M}{\Gamma}\right] .
$$

So, at small $q^{2}$, the value $\epsilon\left(q^{2} ; M, \Gamma\right)$ is large and we cannot cut off the negative component. At $q^{2}<0$, an upper integral in (13) can be calculated with the help of the integration rule (6) and calculation gives the same effect. This effect is a direct consequence of the integration rule (6) and connected with the above-described threshold effect. Thus, the account of the negative component is essential for the case of deep virtual states of UP, that is, far from the peak range. This conclusion arises in any quantum field model with spectral representation of the propagator in form (4) and scalar propagator in BW form (7). It should be noted, however, that the status of the above given evaluations and conclusions crucially depends on the difference between the BW approximation and exact finite propagator (which, unfortunately, is unknown).

\section{Propagator of Vector Unstable Particles}

In this section, the result (10) is applied to determine the structure of vector UP's propagator. Here, we suggest that the function $\rho\left(\mathrm{m}^{2}\right)$ for boson UP (scalar and vector) is universal. Such suggestion is in accordance with the mass redefinition scheme $M^{2}=M_{0}^{2}+\mathfrak{R} \Pi(M)$ and relation $M \Gamma=\mathfrak{I} \Pi(M)$ for the case of both scalar and vector UP. First of all, we demonstrate the consistency of expression (10) for $\rho\left(m^{2}\right)$ and input condition $D(q)=D^{\mathrm{BW}}(q)$ with the help of contour integration, which will be used in further considerations. According to (4) and (10) the propagator of scalar UP can be written as follows:

$$
\begin{aligned}
& D(q) \\
& =\frac{1}{\pi} \int_{-\infty}^{+\infty} \frac{M \Gamma d m^{2}}{\left(q^{2}-m^{2}+i \epsilon\right)\left[\left(m^{2}-M^{2}\right)^{2}+M^{2} \Gamma^{2}\right]} .
\end{aligned}
$$


This expression can be represented in the form

$$
D(q)=-\frac{1}{\pi} \int_{-\infty}^{+\infty} \frac{M \Gamma d m^{2}}{\left(m^{2}-z_{0}\right)\left(m^{2}-z_{+}\right)\left(m^{2}-z_{-}\right)},
$$

where $z_{0}=q^{2}+i \epsilon$ and $z_{ \pm}=M^{2} \pm i M \Gamma$. Analytic continuation of the integrand function in (17), where $m^{2} \rightarrow z$, has three poles $z_{0}, z_{+}$, and $z_{-}$in the complex plane. It decreases as $1 /|z|^{2}$ for $|z| \rightarrow \infty$; that is, it satisfies the condition $|f(z)|<$ $N /|z|^{1+\delta}$ for $|z|>R_{0}$, where $N$ and $\delta$ are positive numbers and $R_{0} \rightarrow \infty$. So, we can apply the method of contour integration and rearrange $D(q)$ as follows:

$$
\begin{aligned}
D(q) & =\mp \frac{M \Gamma}{\pi} \oint_{C_{ \pm}} \frac{d z}{\left(z-z_{0}\right)\left(z-z_{+}\right)\left(z-z_{-}\right)} \\
& =2 \pi i \sum_{k} \operatorname{Res}\left(f(z), z_{k}\right) .
\end{aligned}
$$

In (18) $k$ is number of the poles, $\operatorname{Res}\left(f(z), z_{k}\right)$ is the residue at the pole $z_{k}$, and $C_{ \pm}$is a contour in the upper $\left(C_{+}\right)$or lower $\left(C_{-}\right)$half of the complex $z$-plane. The simplest way to perform the integration is to go along the contour $C_{-}$which encloses only one pole $z_{-}$:

$$
\begin{aligned}
D(q) & =\frac{M \Gamma}{\pi} \oint_{C_{-}} \frac{d z}{\left(z-z_{-}\right)} \frac{1}{\left(z-z_{+}\right)\left(z-z_{0}\right)} \\
& =\frac{2 i M \Gamma}{\left(z_{-}-z_{+}\right)\left(z_{-}-z_{0}\right)}=\frac{1}{q^{2}-M^{2}+i M \Gamma} .
\end{aligned}
$$

In (19), we have used the equality $z_{-}-z_{+}=-2 i M \Gamma$. One can check that the same result follows from the integration along the contour $C_{+}$.

Thus, UP can be described in the framework of two different hierarchical levels_- "fundamental" level, by the integral representations (4), (5), and phenomenological one, by the effective theory after integrating out unobservable mass parameter $m^{2}$ according to (19). In the framework of the effective theory, UP is described by the observed physical values $M$ and $\Gamma$, which can always be defined as a positive quantity. So, at this phenomenological level UP has no explicit tachyonic content which could lead to the abovementioned problems. Instead, we get the term $i M \Gamma$ which describes the instability in a traditional way.

To define the structure of vector propagator, we assume that the spectral function $\rho\left(\mathrm{m}^{2}\right)$ is the same as for a scalar UP. Using the standard vector propagator for a free vector particle with a fixed mass, we get

$$
\begin{aligned}
D_{\mu \nu}(q) & =\frac{1}{\pi} \\
\cdot \int_{-\infty}^{+\infty} \frac{-g_{\mu \nu}+q_{\mu} q_{\nu} /\left(m^{2}-i \epsilon\right)}{q^{2}-m^{2}+i \epsilon} & \frac{M \Gamma d m^{2}}{\left[m^{2}-M^{2}\right]^{2}+M^{2} \Gamma^{2}} .
\end{aligned}
$$

In the term $q_{\mu} q_{\nu} /\left(m^{2}-i \epsilon\right)$ we use the same rule of going around pole as in the denominator $q^{2}-\left(m^{2}-i \epsilon\right)$. The integral in (20) can be evaluated with the help of formula (6); however, it is easier to do it using the method of contour integration. The integration along the lower contour $C_{-}$gives

$$
\begin{aligned}
D_{\mu \nu}(q) & =-\frac{M \Gamma}{\pi} \oint_{C_{-}} \frac{\left(g_{\mu \nu}-q_{\mu} q_{\nu} /(z-i \epsilon)\right) d z}{\left(z-z_{-}\right)\left(z-z_{+}\right)\left(z-z_{0}\right)} \\
& =-2 i M \Gamma \frac{g_{\mu \nu}-q_{\mu} q_{\nu} /\left(z_{-}\right)}{\left(z_{-}-z_{+}\right)\left(z_{-}-z_{0}\right)} \\
& =\frac{-g_{\mu \nu}+q_{\mu} q_{\nu} /\left(M^{2}-i M \Gamma\right)}{q^{2}-M^{2}+i M \Gamma} .
\end{aligned}
$$

One can check that the integration along the upper contour $C_{+}$or with the help of the formula (7) leads to the same result. The expression (21) coincides with the well-known expression for modified BW propagator (3) which satisfies to electromagnetic Ward identity [3].

We should note that both the scalar and vector propagators of UP can be represented in the form with universal complex mass squared:

$$
\begin{aligned}
D(q) & =\frac{1}{q^{2}-M_{P}^{2}}, \\
D_{\mu \nu}(q) & =\frac{-g_{\mu \nu}+q_{\mu} q_{\nu} / M_{P}^{2}}{q^{2}-M_{P}^{2}},
\end{aligned}
$$

where the structure $M_{P}^{2}=M^{2}-i M \Gamma$ usually is called complex-mass definition. This definition is the base element of the so-called complex-mass scheme of calculation $[16,17]$. The dressed propagator of a bosonic UP can be formally obtained from the "free" propagator by the substitution $M_{0}^{2}-$ $i \epsilon \rightarrow M^{2}-i M \Gamma$. So, the infinitesimal value $\epsilon$, which formally defines the rule of going around pole in bare propagator, is an analog of the infinitesimal width of the intermediate state in the framework of the model approach.

\section{Propagator of Spinor Unstable Particles}

The propagator of a free fermion can be represented in two equivalent forms:

$$
\widehat{D}(q)=\frac{1}{\widehat{q}-m+i \epsilon}=\frac{\widehat{q}+m-i \epsilon}{q^{2}-(m-i \epsilon)^{2}} .
$$

According to the abovementioned formal rule for constructing the dressed propagator, we have to make the substitution $m-i \epsilon \rightarrow M-i \Gamma / 2$. Then, the dressed propagator of the spinor UP takes the form (3). Now, we show that the expression (3) can be derived in a more systematic way with the help of the integral representation:

$$
\widehat{D}(q)=\int \frac{\widehat{q}+m-i \epsilon}{q^{2}-(m-i \epsilon)^{2}} \rho(m) d m,
$$

where the integration range is not defined yet. The spectral function $\rho(m)$ for fermions differs from the bosonic one, because of another parametrization $M(q)=M_{0}+\Re \Sigma(q)$ and 
$\Gamma(q)=\Im \Sigma(q)$. The spectral function for the case of the spinor UP is as follows:

$$
\begin{aligned}
\rho(m) & =\frac{1}{\pi} \frac{\Gamma / 2}{[m-M]^{2}+\Gamma^{2} / 4} \\
& =\frac{1}{\pi} \frac{\Gamma / 2}{\left(m-M_{-}\right)\left(m-M_{+}\right)},
\end{aligned}
$$

where $M_{ \pm}=M \pm i \Gamma / 2$. The main difference between boson and spinor cases is a presence of the linear term $m$ instead the quadratic one $\mathrm{m}^{2}$, which is defined at the whole real axis $m^{2} \in(-\infty,+\infty)$. Here, we consider a straightforward relation between the bosonic parameter range and spinor one. Thus, we have two intervals, namely, $(+i \infty, i 0 ; 0, \infty)$ and $(-i \infty, i 0 ; 0, \infty)$, for the value $m$. In the method of contour integration the signs \pm correspond to integration along the contours $C_{ \pm}$, which enclose the first or fourth quadrants of the complex plane. Then, from (24) and (25) it follows that

$$
\widehat{D}_{ \pm}(q)= \pm \frac{\Gamma}{2 \pi} \int_{C_{ \pm}} \frac{(\widehat{q}+z) d z}{\left(z^{2}-z_{0}^{2}\right)\left(z-z_{-}\right)\left(z-z_{+}\right)}
$$

where $z_{0}^{2}=q^{2}+i \epsilon, z_{ \pm}=M_{ \pm}$, and $C_{ \pm}$are the above described contours. By simple and straightforward calculations we can see that the correct result follows from the integration along the contour $C_{-}$, while the integration along the $C_{+}$leads to nonphysical result. This is likely caused by the presence of the branch point $z_{0}^{2}$ in the first quadrant. From (26) it follows that

$$
\begin{aligned}
\widehat{D}_{-}(q) & =-\frac{\Gamma}{2 \pi} \int_{C_{-}} \frac{d z}{z-z_{-}} \frac{\widehat{q}+z}{\left(z^{2}-z_{0}^{2}\right)\left(z-z_{+}\right)} \\
& =-i \Gamma(q) \frac{\widehat{q}+z_{-}}{\left(z_{-}^{2}-z_{0}^{2}\right)\left(z_{-}-z_{+}\right)} \\
& =\frac{\widehat{q}+M-i \Gamma / 2}{q^{2}-(M-i \Gamma / 2)^{2}} .
\end{aligned}
$$

The last expression in (27) coincides with the corresponding expression in (3). The spinor complex-mass definition differs from the bosonic one; however, it has similar pole-type complex structure. Then, the pole definition of the mass and width of the spinor UP is $M_{P}=M_{\rho}-i \Gamma_{\rho} / 2$ in our consideration.

\section{Complex-Mass Scheme in Effective Theory}

The main result of the previous two sections is the modified BW expressions (3) derived in analytical way. We have showed also the connection between scalar, vector, and spinor propagator with the help of the spectral approach. Note that the analogous connection takes place for the case of free fields, that is, for the stable particle approximation [37]:

$$
\begin{aligned}
D_{i k}(x) & =\left(g_{i k}+\frac{1}{M^{2}} \frac{\partial^{2}}{\partial^{i} \partial^{k}}\right) D(x) \\
& =\frac{1}{(2 \pi)^{4}} \int \frac{\left(g_{i k}-k_{i} k_{k} / M^{2}\right) e^{-i k x}}{k^{2}-M^{2}} d k,
\end{aligned}
$$

$$
\begin{aligned}
\widehat{D}(x) & =(i \widehat{\partial}+M) D(x) \\
& =\frac{1}{(2 \pi)^{4}} \int \frac{\widehat{k}+M}{k^{2}-M^{2}} e^{-i k x} d k .
\end{aligned}
$$

In (28) the value $M^{2}=M_{0}^{2}-i \epsilon$ and $D(x)$ is scalar casual function, that is, propagator in coordinate representation for free scalar field $\Phi(x)$, which satisfies to Klein-Gordon equation:

$$
\left(\partial_{k} \partial^{k}-M^{2}\right) \Phi(x)=0
$$

We have derived just the same expressions for the case of dressed propagators in the momentum representation, that is, for complex mass $M=M_{P}$, which should be inserted into Lagrangian (vertexes, $\sin ^{2} \theta_{W}$, etc.) and into motion equation (29). In such way, we have come to the so-called complexmass scheme of calculation [16-18], which is realized at Lagrangian level of effective theory. It should be noted that free casual functions $D_{i k}(x)$ and $\widehat{D}(x)$, which are defined by (28), in analogy with scalar one can be represented as vacuum expectation value of chronological field operator product [37]:

$$
\begin{aligned}
D_{i k}(x-y) & =i\left\langle 0\left|T\left(\phi_{i}(x) \phi_{k}(y)\right)\right| 0\right\rangle, \\
\widehat{D}(x-y) & =i\langle 0|T(\psi(x) \bar{\psi}(y))| 0\rangle .
\end{aligned}
$$

As it was shown in Section 2, the scalar field function can be redefined according to (5) with weight function $\omega\left(m^{2}\right)= \pm \sqrt{\rho\left(m^{2}\right)}$ which leads to BW propagator. The same redefinition can be done for the case of vector and spinor UP, which provides the validity of (30). So, we have effective theory which has some principal properties of fundamental quantum field theory. As was noted in Section 3, UP can be described at two hierarchical levels, fundamental one and phenomenological one (after integrating out unobservable mass parameter $\mathrm{m}^{2}$ ). In the case under consideration, the phenomenological approach corresponds to the effective theory, which can be represented at lowest order by the Lagrangian with complex masses. The effective field function, which describes UP, is defined in a correspondence with the conventional definition of Green's function.

The problems of renormalization procedure in the effective theory arise at next-to-leading order. To date there is no fully established treatment of UP within perturbation theory, although many solutions have been proposed [18]. For instance, the unitarity in scalar field theories was studied within the framework of the CMS [18] at one-loop approximation. Evidently, the effective field function of UP is formed by self-energy contribution at fundamental level and contains corresponding information about mass and width of UP. In the case under consideration, this information is included into spectral function $\rho\left(\mathrm{m}^{2} ; M, \Gamma\right)$. So, we have to avoid double counting of self-energy contribution in the calculations at loop level of the effective theory. Just this contribution stipulates the divergence of renormalization 
constant at threshold $M \rightarrow 2 M_{V}$, where the vectors are in the self-energy loop [20]. Thus, we do not deal with the conventional TS in the framework of the effective theory. As was shown in Section 2, the problem of TS appears at the leading order, and its nature differs from the conventional one. It should be noted, however, that mathematically strict status of our consideration is due to appearance of the negative spectral component which has no clear physical meaning.

\section{Conclusions}

The definitions of the mass and width of UP, as a rule, are closely connected with the construction of the dressed propagators. It was underlined in this work that traditional approaches, which are based on the Dyson procedure and spectral representation, have formally crucial peculiarities. We have considered the structure of the propagators of UP in the phenomenological approach which is based on the spectral representation. The spectral function describes the distribution of continuous (indefinite, smeared) mass parameter, contains a principal information concerning UP, and defines a spectral structure of the propagators.

In this work, we have analyzed a special case of the spectral function which follows from matching the model and standard scalar BW propagator. This function contains the parameters $M, \Gamma$ and mass variable $m^{2}$ proven to be in the interval $(-\infty,+\infty)$. So, the variable $m$ can be imaginary; however, such states have no explicit physical content. It was shown that contribution of the negative component to the full propagator is significant for the deep virtual states. In the framework of this approach we get vector and spinor propagators with the well-known modified BW structure. This structure provides the gauge invariant description and explicitly leads to the complex-mass definition. The $q$ dependence of the UP mass and width can be introduced into the function $\rho\left(m^{2} ; \Gamma(q), M(q)\right)$ without the loss of the generality. We formulated some problematical aspects of the propagators construction which require an additional analysis.

\section{Conflict of Interests}

The author declares that there is no conflict of interests regarding the publication of this paper.

\section{Acknowledgment}

The work has been supported by Southern Federal University Grant no. 213.01-2014/013-VG.

\section{References}

[1] A. Sirlin, "Observations concerning mass renormalization in the electroweak theory," Physics Letters B, vol. 267, no. 2, pp. 240-242, 1991.

[2] S. Willenbrock and G. Valencia, "On the definition of the $Z$ boson mass," Physics Letters B, vol. 259, no. 3, pp. 373-376, 1991.
[3] G. Lopez Castro, J. L. M. Lucio, and J. Pestieau, " $W^{ \pm}$propagator on the resonance region," Modern Physics Letters A, vol. 6, no. 40, pp. 3679-3682, 1991.

[4] R. G. Stuart, "Gauge invariance, analyticity and physical observables at the $Z^{0}$ resonance," Physics Letters, Section B: Nuclear, Elementary Particle and High-Energy Physics, vol. 262, no. 1, pp. 113-119, 1991.

[5] A. Leike, T. Riemann, and J. Rose, " $S$-matrix approach to the $Z$ line shape," Physics Letters B, vol. 273, no. 4, pp. 513-518, 1991.

[6] B. A. Kniehl, C. P. Palisoc, and A. Sirlin, "Higgs-boson production and decay close to thresholds," Nuclear Physics B, vol. 591, no. 1-2, pp. 296-310, 2000.

[7] J. L. M. Lucio and J. Pestieau, "Resonance propagation and threshold singularities," Modern Physics Letters A, vol. 16, no. 20, pp. 1315-1320, 2001.

[8] M. Faisst, J. H. Kühn, and O. Veretin, "Pole- versus MS-mass definitions in the electroweak theory," Physics Letters B, vol. 589, no. 1-2, pp. 35-38, 2004.

[9] A. R. Bohm and Y. Sato, "Relativistic resonances: their masses, widths, lifetimes, superposition, and causal evolution," Physical Review D, vol. 71, no. 8, pp. 1-22, 2005.

[10] Y. Zhou, "Gauge dependence of on-shell and pole mass renormalization prescriptions," Communications in Theoretical Physics, vol. 49, no. 5, pp. 1249-1260, 2008.

[11] G. Cacciapaglia, A. Deandrea, and S. De Curtis, "Nearby resonances beyond the Breit-Wigner approximation," Physics Letters B, vol. 682, no. 1, pp. 43-49, 2009.

[12] A. R. Bohm and N. L. Harshman, "On the mass and width of the Z-boson and other relativistic quasistable particles," Nuclear Physics B, vol. 581, no. 1-2, pp. 91-115, 2000.

[13] M. Nowakowski and A. Pilaftsis, "On gauge invariance of BreitWigner propagators," Zeitschrift für Physik C, vol. 60, no. 1, pp. 121-125, 1993.

[14] G. López Castro, J. L. Lucio M, and J. Pestieau, "Remarks on the $W$ propagator at the resonance," International Journal of Modern Physics A, vol. 11, no. 3, pp. 563-570, 1996.

[15] J. Liu, "Particle-width effect on CP-violating asymmetries," Physical Review D, vol. 47, no. 5, pp. R1741-R1743, 1993.

[16] A. Denner, S. Dittmaier, M. Roth, and D. Wackeroth, "Predictions for all process $e^{+} e^{-} \rightarrow$ fermions $+\gamma$," Nuclear Physics B, vol. 560, no. 1-3, pp. 33-65, 1999.

[17] A. Denner, S. Dittmaier, M. Roth, and L. H. Wieders, "Electroweak corrections to charged-current $e^{+} e^{-} \rightarrow 4$ fermion processes: technical details and further results," Nuclear Physics B, vol. 724, no. 1-2, pp. 247-294, 2005.

[18] A. Denner and J.-N. Lang, "The complex-mass scheme and unitarity in perturbative quantum field theory," The European Physical Journal C, vol. 75, no. 8, article 377, 2015.

[19] D. Djukanovic, E. Epelbaum, J. Gegelia, H. Krebs, and U.-G. Meißner, "Complex-mass renormalization in hadronic EFT: applicability at two-loop order," The European Physical Journal A, vol. 51, no. 8, article 101, 2015.

[20] B. A. Kniehl and A. Sirlin, "Pole mass, width, and propagators of unstable fermions," Physical Review D, vol. 77, no. 11, Article ID 116012, 2008.

[21] L. De Cruz, T. Vrancx, P. Vancraeyveld, and J. Ryckebusch, "Bayesian inference of the resonance content of $p\left(\gamma, K^{+}\right) \Lambda$," Physical Review Letters, vol. 108, no. 18, Article ID 182002, 2012.

[22] S. Ceci, M. Korolija, and B. Zauner, "Model-independent extraction of the pole and Breit-Wigner resonance parameters," Physical Review Letters, vol. 111, no. 11, Article ID 112004, 2013. 
[23] S. Ceci, M. Vuksic, and B. Zauner, "Breit-Wigner phase is a fundamentalproperty of a resonance," http://arxiv.org/abs/1408 .2437 .

[24] H. Lehmann, "Über Eigenschaften von Ausbreitungsfunktionen und Renormierungskonstanten quantisierter Felder," Il Nuovo Cimento, vol. 11, pp. 342-357, 1954.

[25] M. Gell-Mann and F. E. Low, "Quantum electrodynamics at small distances," Physical Review, vol. 95, no. 5, pp. 1300-1312, 1954.

[26] G. Kallen, "On the defenition of the renormalization constants in quantum electrodynamics," Helvetica Physica Acta, vol. 25, pp. 417-435, 1957.

[27] P. T. Matthews and A. Salam, "Relativistic field theory of unstable particles," Physical Review, vol. 112, no. 1, pp. 283-287, 1958.

[28] J. Schwinger, "Field theory of unstable particles," Annals of Physics, vol. 9, no. 2, pp. 169-193, 1960.

[29] R. Jacob and R. G. Sachs, "Mass and lifetime of unstable particles," Physical Review Letters, vol. 121, no. 1, pp. 350-356, 1961.

[30] O. W. Greenberg, "Generalized free fields and models of local field theory," Annals of Physics, vol. 16, no. 2, pp. 158-176, 1961.

[31] A. L. Licht, "A generalized asymptotic condition. I," Annals of Physics, vol. 34, no. 1, pp. 161-186, 1965.

[32] Y. Burnier and A. Rothkopf, "Bayesian approach to spectral function reconstruction for Euclidean quantum field theories," Physical Review Letters, vol. 111, no. 18, Article ID 182003, 2013.

[33] D. Dudal, O. Oliveira, and P. J. Silva, "Källén-Lehmann spectroscopy for (un)physical degrees of freedom," Physical Review D, vol. 89, no. 1, Article ID 014010, 2014.

[34] M. F. M. Lutz, E. E. Kolomeitsev, and C. L. Korpa, "Spectral representation fo $u$ - and $t$-channel exchange processes in a partial-wave decomposition," Physical Review D, vol. 92, no. 1, Article ID 016003, 18 pages, 2015.

[35] V. I. Kuksa, "Finite-width effects in the model of unstable particles with a smeared mass," International Journal of Modern Physics A, vol. 24, no. 6, pp. 1185-1205, 2009.

[36] V. I. Kuksa, "Unstable states in quantum theory," Physics of Particles and Nuclei, vol. 45, no. 3, pp. 568-608, 2014.

[37] N. N. Bogolubov and D. V. Shirkov, An Introduction to Quantum Field Theory, Nauka, Moscow, Russia, 1976. 

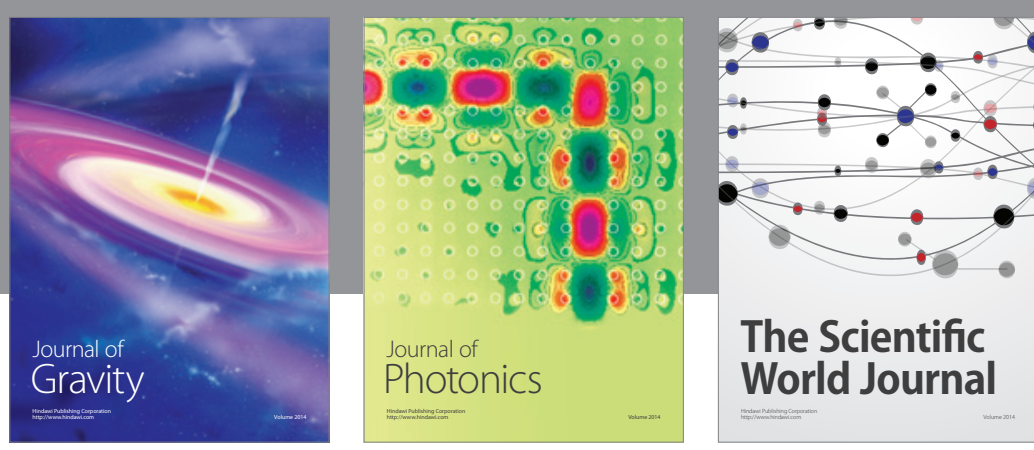

The Scientific World Journal
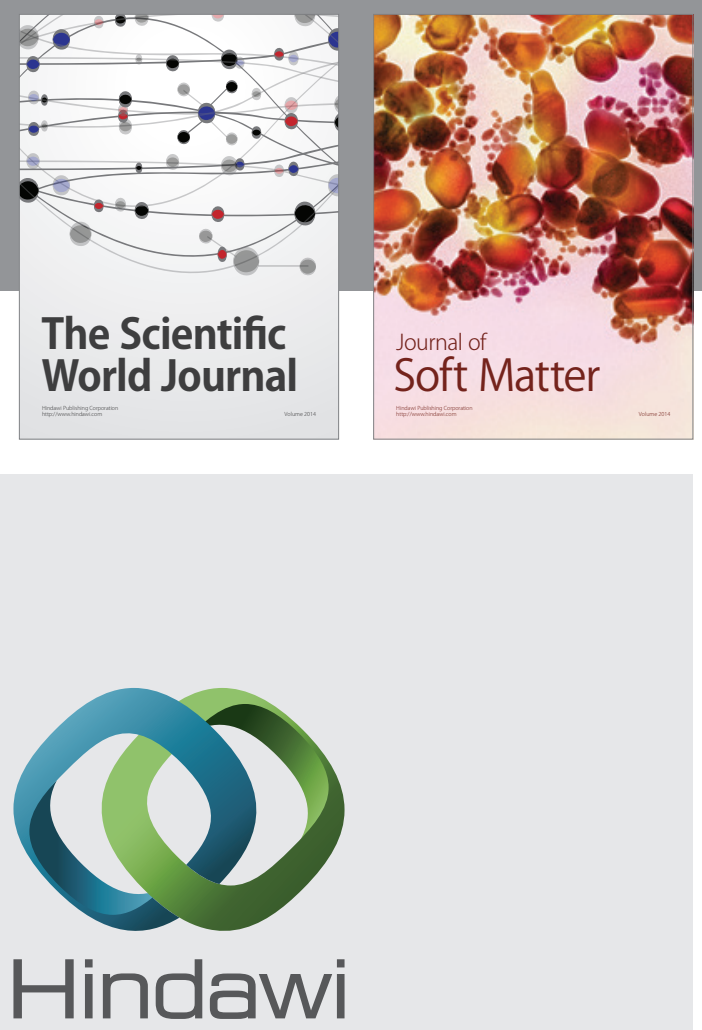

Submit your manuscripts at

http://www.hindawi.com

nternational Journal of

Statistical Mechanics
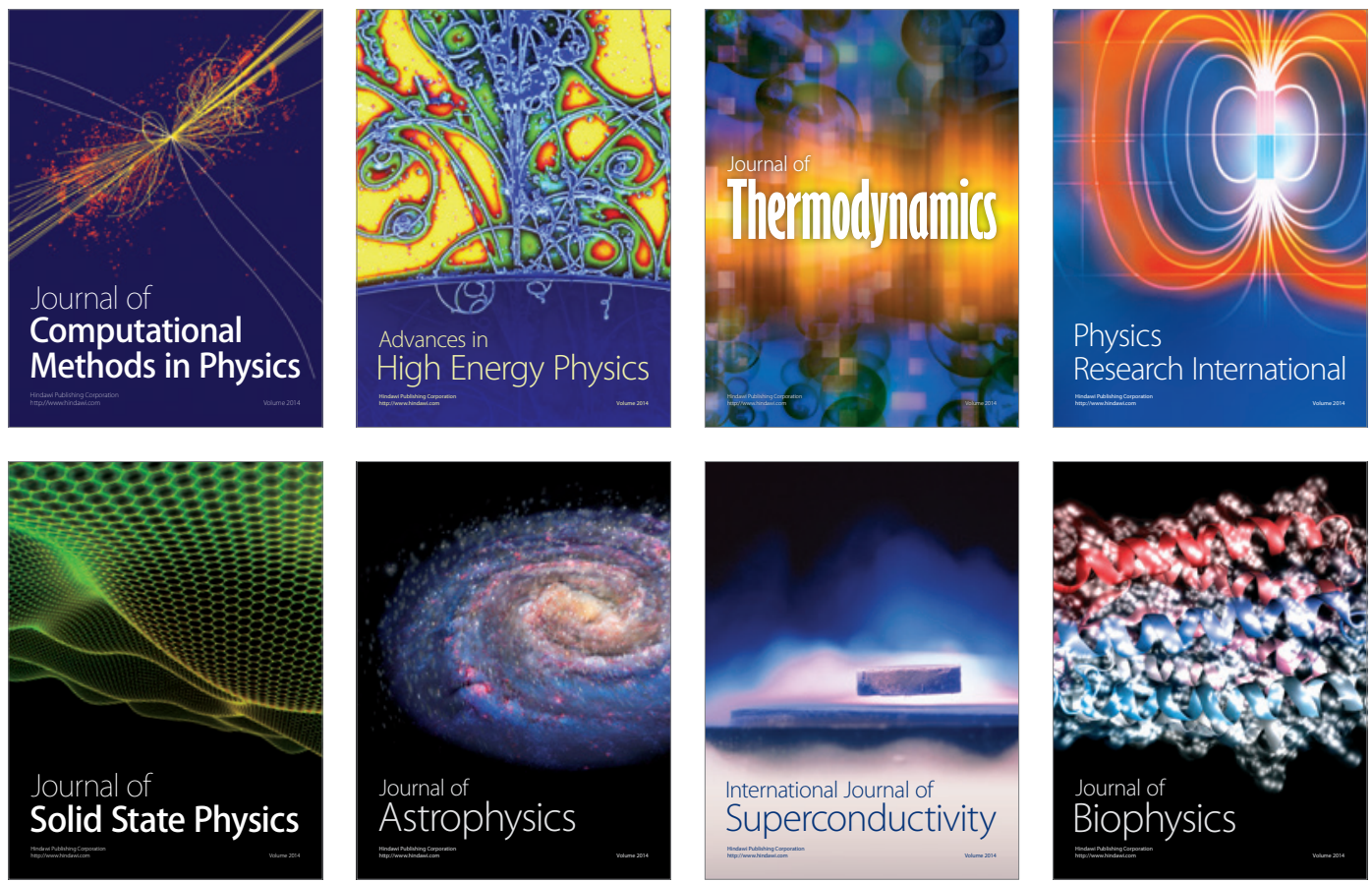
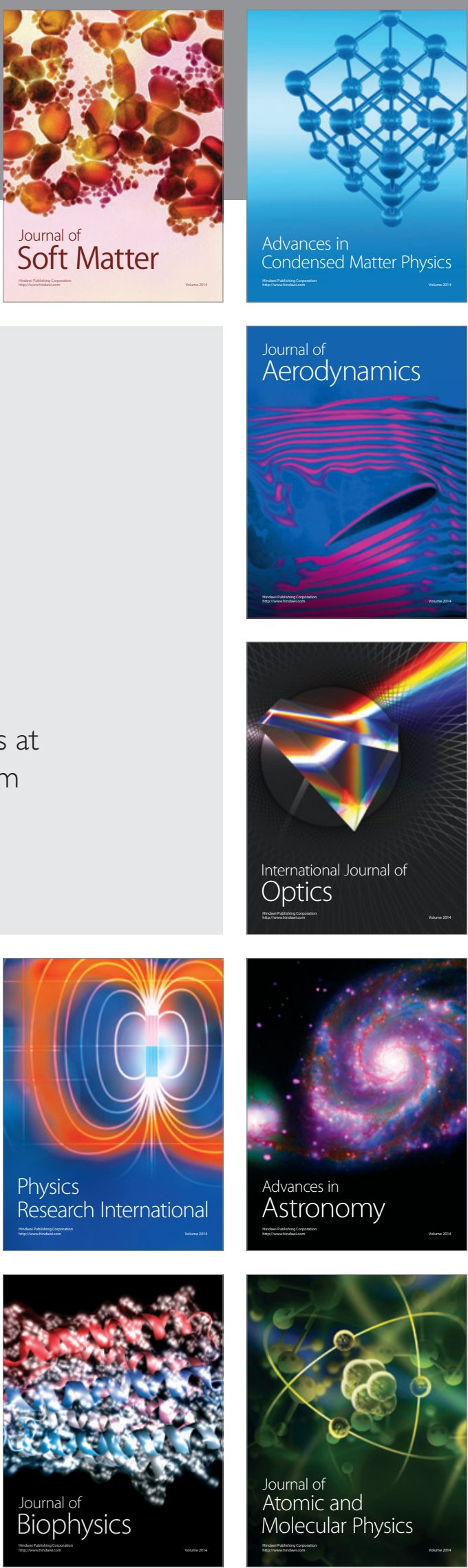